
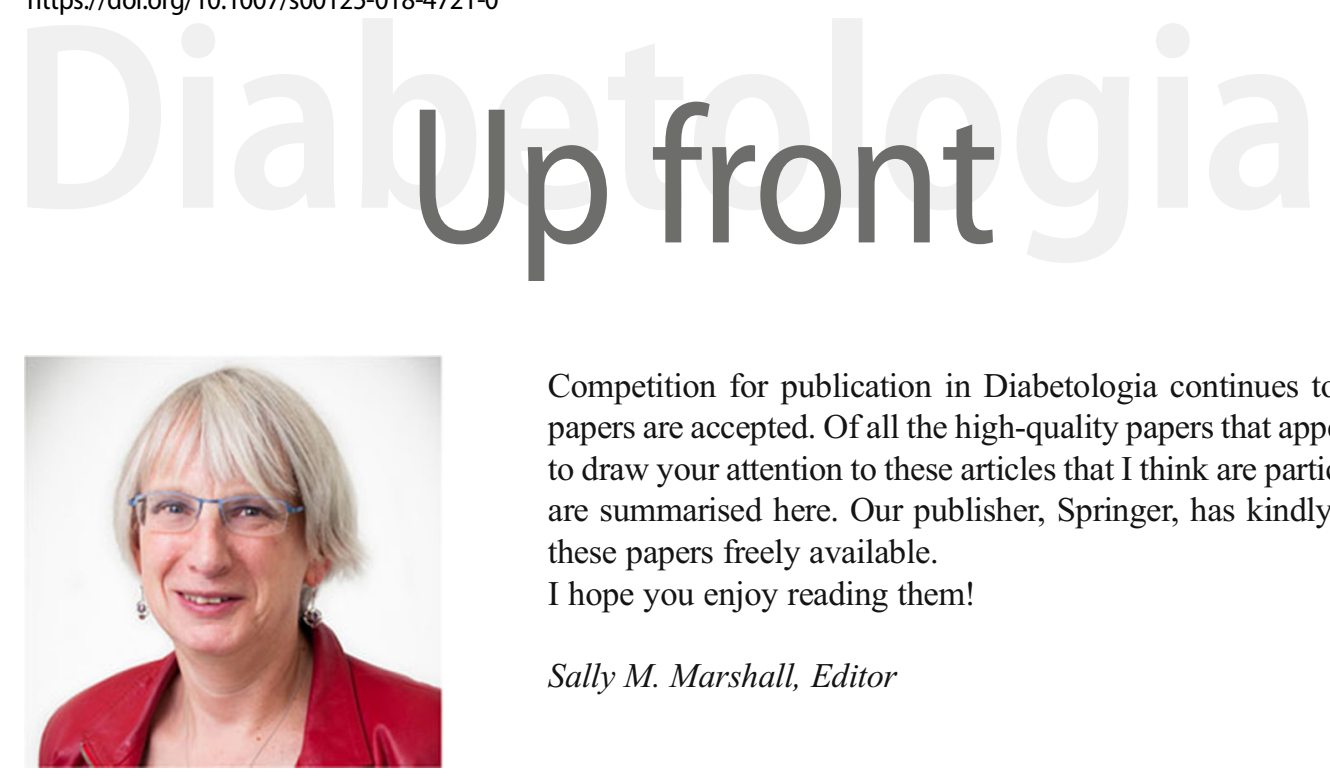

Competition for publication in Diabetologia continues to grow, and less than $20 \%$ of papers are accepted. Of all the high-quality papers that appear in this month's issue I want to draw your attention to these articles that I think are particularly interesting. The articles are summarised here. Our publisher, Springer, has kindly made the full text of each of these papers freely available.

I hope you enjoy reading them!

Sally M. Marshall, Editor

\section{The bark giving diabetes therapy some bite: the SGLT} inhibitors

This issue features a special series of reviews that focus on the newest class of glucose-lowering agents, the sodium-glucose cotransporter (SGLT) inhibitors. Rieg and Vallon (https://doi. org/10.1007/s00125-018-4654-7) begin the series by tracing the development of the SGLT inhibitor class of drugs, including SGLT1 inhibitors, SGLT2 inhibitors and dual inhibitors. Wright and colleagues (https://doi.org/10.1007/ s00125-018-4656-5) go on to discuss the mechanisms of actions of these drugs; they explain that SGLT1 and SGLT2 (and GLUT2) are key players in renal glucose transport and describe how inhibition of either SGLT2 or SGLT1 promotes glucose excretion in the urine. However, as discussed by Thomas and Cherney (https://doi.org/10.1007/s00125-0184669-0), SGLT inhibitors not only affect glucose metabolism, but also body weight, renal function and blood pressure in type 2 diabetes. In terms of their pleiotropic actions, the most striking results so far come from SGLT2 inhibitor studies investigating the cardiovascular effects of these drugs. In their review, Verma and McMurray (https:// doi.org/10.1007/s00125-018-4670-7) outline the proposed mechanisms underpinning the unprecedented benefit of reduced cardiovascular disease risk with SGLT2 inhibitor use, observed in people with type 2 diabetes with established cardiovascular disease or multiple cardiovascular risk factors. Despite their glucose-lowering ability, pleiotropic effects and potential cardioprotective outcomes, the place of SGLT2 inhibitors in the management of type 2 diabetes is still hotly debated. To explain why, Lupsa and Inzucchi (https:// doi.org/10.1007/s00125-018-4663-6) review the benefits and adverse effects of SGLT2 inhibitors approved for use in the USA and Europe in individuals with type 2 diabetes. What about individuals with type 1 diabetes? Research is much sparser in this area but McCrimmon and Henry (https://doi. org/10.1007/s00125-018-4671-6) discuss the results of two recent 24 week Phase III randomised controlled clinical trials, inTandem 3 and DEPICT-1, which studied sotagliflozin (a dual SGLT1/2 inhibitor) and dapagliflozin (an SGLT2 inhibitor), respectively. Wanner and Marx (https://doi.org/10.1007/s00125-018-4678-z) conclude the series by discussing SGLT2 inhibitors in the context of the future of diabetes therapy. They also discuss the effects of SGLT2 inhibitors on other chronic diseases and outline future treatment strategies. This review set is accompanied by an editorial by Sally Marshall (https://doi.org/10.1007/ s00125-018-4673-4).

Sex differences in the association between diabetes and cancer: a systematic review and meta-analysis of 121 cohorts including $\mathbf{2 0}$ million individuals and one million events

Toshiaki Ohkuma, Sanne A. E. Peters, Mark Woodward

In several systematic reviews and meta-analyses, diabetes has been associated with the risk of all-site and some site-specific cancers. However, there has been no systematic overview of the evidence available on sex differences in the association between diabetes and cancer. In this issue, Ohkuma et al (https://doi.org/10.1007/s00125-018-4664-5) report that diabetes was associated with a higher risk of all-site cancer 
in both sexes, but there was a $\sim 6 \%$ greater risk in women compared with men. Diabetes was also associated with several site-specific cancers and conferred a significantly greater excess risk in women than men for oral, stomach and kidney cancer, and for leukaemia, but a lower excess risk for liver cancer. These findings indicate the importance of a sexspecific approach to analysis of the role of diabetes for cancer prevention and treatment.

Gluten intake and risk of type 2 diabetes in three large prospective cohort studies of US men and women

Geng Zong, Benjamin Lebwohl, Frank B. Hu, Laura Sampson, Lauren W. Dougherty, Walter C. Willett, Andrew T. Chan, Qi Sun

Avoidance of gluten intake is crucial for the management of coeliac disease, in which gluten triggers an autoimmune response. However, adoption of a gluten-free diet among people without apparent gluten-related disorders in the USA and many other countries has become increasingly popular, with the belief that eating a gluten-free diet is associated with health benefits. Despite this perception, evidence is lacking to support or refute the belief that avoidance of gluten is associated with cardiometabolic health benefits in populations without coeliac disease. To fill this knowledge gap, in this issue, Zong, Lebwohl et al (https://doi.org/10.1007/s00125-018-4697-9) report findings from a large-scale analysis in three long-running cohorts of US men and women. They found an inverse association between gluten intake and risk of type 2 diabetes. This association was independent of established diabetes risk factors and appeared to be stronger when added bran intake was also higher. These results suggest that gluten intake is unlikely to exert adverse effects on diabetes risk and that the avoidance of gluten intake, often at the price of reducing fibre intake, should not be recommended for diabetes prevention.

\section{Programming of central and peripheral insulin resistance by low birthweight and postnatal catch-up growth in male mice}

Lindsey M. Berends, Laura Dearden, Yi Chun L. Tung, Peter Voshol, Denise S. Fernandez-Twinn, Susan E. Ozanne

Low birthweight followed by accelerated postnatal growth is associated with increased risk of type 2 diabetes. It is well established that this is, at least in part, due to programmed peripheral insulin resistance. However, it is unclear if a suboptimal early-life environment also programs central insulin resistance. In this issue, Berends, Dearden et al (https://doi. org/10.1007/s00125-018-4694-z) use a mouse model of dietinduced intra-uterine growth restriction (IUGR) followed by accelerated postnatal catch-up growth to show that a suboptimal early-life environment causes insulin to be less effective at signalling to the brain to reduce food intake in later life. Central insulin resistance in IUGR followed by accelerated postnatal growth was shown to be related to altered expression of insulin-signalling components in the brain, as compared with control offspring born to dams fed a control diet. These findings indicate that promoting accelerated growth in offspring born small for gestational age could have negative effects on long-term metabolic health and that central insulin resistance may contribute to this phenomenon. If extrapolated to humans, these findings also suggest that individuals exposed to a suboptimal early-life environment may be less responsive to both lifestyle and pharmaceutical interventions for metabolic improvements in type 2 diabetes

\section{A novel dual PPAR- $\gamma$ agonist/sEH inhibitor treats diabetic complications in a rat model of type 2 diabetes}

Md Abdul Hye Khan, Lauren Kolb, Melissa Skibba, Markus Hartmann, René Blöcher, Ewgenij Proschak, John D. Imig

Despite significant progress in diabetes management, approximately $50 \%$ of people with type 2 diabetes fail to achieve therapeutic goals. Consequently, rates of diabetes-associated morbidity and mortality are high, mainly due to complications such as cardiovascular, liver and kidney disease. Poor clinical outcomes with current therapies for diabetes are associated with their lack of ability to simultaneously lower blood glucose and treat comorbidities. Consequently, the majority of patients with type 2 diabetes with a comorbid condition require a multi-drug approach to treatment. In this issue, Hye Khan et al (https://doi.org/10.1007/s00125-018-4685-0) report data from a study in which they developed a novel dual-acting molecule, RB394, that concurrently acts as an inhibitor of soluble epoxide hydrolase (sEH) and an activator of peroxisome proliferator-activated receptor- $\gamma(\operatorname{PPAR}-\gamma)$. In rat models of the metabolic syndrome and type 2 diabetes, which are associated with comorbid cardiovascular, liver and kidney disease, the authors demonstrated that RB394 not only ameliorated type 2 diabetes and its comorbid conditions, but also treated multiple diabetic complications, including diabetic nephropathy and liver injury. The authors conclude that RB394 is a promising molecule with the potential for development into a therapeutic agent for the metabolic syndrome, type 2 diabetes and associated complications.

All text supplied by the authors. 